\title{
Neoadjuvant therapy of gastric cancer with the human monoclonal IgM antibody SC-1: Impact on the immune system
}

\author{
ULRICH BEUTNER $^{1 *}$, UDO LORENZ $^{1 *}$, BERTRAM ILLERT $^{1}$, LORETTA ROTT $^{1}$, WOLFGANG TIMMERMANN $^{1}$, \\ H. PETER VOLLMERS ${ }^{2}$, HANS KONRAD MÜLLER-HERMELINK ${ }^{2}$, ARNULF THIEDE ${ }^{1}$ and KARIN ULRICHS ${ }^{1}$ \\ ${ }^{1}$ Department of Surgery I, Centre for Operative Medicine, University of Würzburg, Oberdürrbacher Str. 6; \\ ${ }^{2}$ Department of Pathology, University of Würzburg, Josef-Schneider-Str. 2, D-97080 Würzburg, Germany
}

Received March 19, 2007; Accepted June 14, 2007

\begin{abstract}
Adjuvant therapies for minimal residual disease are a promising approach to improve the poor survival rates after surgery of gastric tumors. A pilot study of a neoadjuvant therapy was performed using a human monoclonal IgM antibody (SC-1) specifically inducing apoptosis in signet ring cell stomach carcinomas. However, scarce information exists on how such a treatment affects the immune system, in particular what are the effects of apoptosis induction and infusion of large amounts of IgM. Thus, the leukocyte composition (CD3, CD4, CD8, CD19, CD16+56, CD14) and several cytokines (TNF- $\alpha$, IL6, IL12, IFN- $\gamma$, GM-CSF, Neopterin) before and after SC-1 application were measured and compared to results of patients that underwent surgical removal of gastric carcinoma without antibody treatment. After SC-1 application, an increase in TNF- $\alpha$ and a decrease of lymphocytes and $\mathrm{CD}^{+} \mathrm{T}$-cells but in the range obtained in healthy individuals was observed before surgery. After surgery, the IL6 levels increased and the TNF- $\alpha$ levels remained at the elevated level. Furthermore, there was a significant drop in lymphocytes and $\mathrm{CD}^{+} \mathrm{T}$-cells. These effects were due to the surgical treatment. Other parameters did not show significant changes. It seems that the application of an apoptosis-inducing antibody prior to surgery of gastric tumors has mild if any effect on the immune system. Therefore, from an immunological point of view, the treatment with this monoclonal antibody is extremely safe.
\end{abstract}

\section{Introduction}

In recent years, many monoclonal antibodies have been approved for clinical tumor therapy. Most of the antibodies

Correspondence to: Dr Udo Lorenz, Department of Surgery I, Centre for Operative Medicine, University of Würzburg, Oberdürrbacher Str. 6, D-97080 Würzburg, Germany

E-mail: u.lorenz@mail.uni-wuerzburg.de

${ }^{*}$ Contributed equally

Key words: antibody therapy, gastric cancer, monoclonal, immune system, SC-1 are directed against single cell tumors such as leukaemia but rarely against large solid tumors $(1,2)$. While the treatment of large solid tumors with monoclonal antibodies alone will be restricted to very few cases, the use of monoclonal antibodies in the adjuvant therapy of solid tumors before or after conventional treatment, like surgery, seems to be very promising. After surgical resection of tumors, single tumor cells can remain (minimal residual disease) or the tumor could have already formed micrometastases leading to a relapse of the disease (3-5). Minimal residual disease and micrometastases are an ideal target for antibody-mediated therapy. Between 1997 and 2000, a clinical pilot study was performed at the University Hospital Würzburg to evaluate such a combined therapy of surgical removal and antibody treatment (6).

A monoclonal antibody named SC-1 was used that is highly specific for signet ring cell stomach carcinomas (7). It binds to a tumor specific carbohydrate epitope on the decayaccelerating factor (DAF, CD55) (8); however, this was not known at the time the pilot study was performed. The monoclonal antibody SC-1 is an IgM of human origin most likely derived from B1-cells. B1-cells [opposed to the better-known B2 (B-) cells] produce large amounts of mainly IgM antibodies without any apparent immunization. The anti-blood group antibodies are the best-known example of B1-derived immunoglobulins.

The most intriguing property of the SC-1 antibody is the ability to induce apoptosis simply by binding to the epitope with no help from factors such as complement or by cells like in the antibody-dependent cellular cytotoxicity (ADCC) $(9,10)$. Thus, the SC-1 antibody is a good candidate for clinical application: it is highly specific, it induces apoptosis in the targeted tumor cells and the human B1-cell origin makes the induction of serum disease or other anti-antibody reactions extremely unlikely.

For the pilot study, binding of the SC-1 antibody to carcinoma cells in biopsies from stomach cancer patients was analyzed. Patients who were positive for the SC-1 epitope received one single dose of 20-30 mg purified SC-1 IgM i.v. 1-2 days prior to the surgical removal of the tumor (6). In most cases of SC-1 treatment, apoptosis was observed in the tumor tissue after surgery (6). Besides the effectiveness of the SC-1 antibody, we were also concerned about the safety of the treatment. Therefore, we investigated whether the 
immune system is affected by the antibody infusion itself and whether we could detect a difference in the immune system in patients with or without SC-1 treatment 7 and 14 days after surgery. We were particularly interested whether the application of the antibody itself has any detrimental effects and whether the antibody treatment leads either to an aberrant activation of the immune system or even to an immunosuppression, which might put the patients at risk post-operation.

To evaluate the immune status of the patients during the therapy, the leukocyte composition was determined by flow cytometry and soluble serum factors were determined by ELISA. We assessed the fraction of lymphocytes and monocytes among the leukocytes and the fraction of T-cells $\left(\mathrm{CD}^{+}\right)$, B-cells (CD19+) and NK-cells $\left(\mathrm{CD} 16 / 56^{+}\right)$among the lymphocytes. The T-cells were further subdivided in helper T-cells $\left(\mathrm{CD}^{+}\right)$and cytotoxic T-cells $\left(\mathrm{CD}^{+}\right)$and the $\mathrm{CD} 4 / \mathrm{CD} 8$ ratio was calculated. Finally, the expression level of HLA-DR on monocytes was determined. Reduced expression of HLA-DR on monocytes has been shown to be an early indicator of strong inflammation such as SIRS or sepsis $(11,12)$.

In the blood serum, the amount of the inflammatory cytokines TNF- $\alpha$, IL6, IL12, IFN- $\gamma$ and GM-CSF was measured as well as the metabolite neopterin. Neopterin is excreted exclusively by activated macrophages and is generated from GTP by the enzyme GTP-cyclohydrolase I $(13,14)$.

\section{Materials and methods}

Pilot study. Between 1997 and 2000, a pilot study using the human monoclonal antibody SC-1 was performed in the Department of Surgery at the University Hospital Würzburg, Germany (6). Gastric cancer biopsies were tested for binding of SC-1. Patients positive for binding were infused with 20$30 \mathrm{mg} \mathrm{SC}-1$ antibody $24-48 \mathrm{~h}$ before surgery. Eighteen patients were analyzed by flow cytometry and 11 patients for serum factors by ELISA. The details of these patients (group S) are summarized in Table I. Tumor stages of patients are according to UICC for R0 resection (15). The results were compared to data obtained from patients with gastric cancer without antibody treatment (group N) (Table I). For curative resection all patients underwent total gastrectomy and lymphadenectomy (D2) with Roux-en-Y gastrojejunostomy. The local ethics committee approved the study and all patients gave informed consent to their participation. For the flow cytometric analysis, additional data were obtained from apparently healthy volunteers (group R) (Table I). Informed consent for blood sampling was obtained from all individuals. The blood samples were taken preoperatively, 7 and 14 days after surgery. Additional blood samples were taken from patients in group $\mathrm{S}$ preoperatively $24 \mathrm{~h}$ after delivery of the SC-1 antibody.

Serum factors. Blood was collected in serum S-Monovettes (Sarstedt, Nümbrecht, Germany), centrifuged and serum supernatant was stored at $-70^{\circ} \mathrm{C}$ until samples were analyzed for serum factors using commercial ELISA or EIA kits. The following factors were measured: interferon- $\gamma$ (IFN- $\gamma$, EASIA, Biosource, Nivelles, Belgium), tumor necrosis factor- $\alpha$ (TNF- $\alpha$, EASIA, Biosource), interleukin 12, p70 subunit (IL12p70 Cytoscreen, Biosource), interleukin 6 (IL6 EASIA, Biosource), neopterin (Brahms, Hennigsdorf,
Table I. Patient characteristics.

\begin{tabular}{|c|c|c|c|c|}
\hline & \multicolumn{2}{|c|}{$\begin{array}{c}\text { Group S } \\
\text { SC-1 treatment }\end{array}$} & \multirow{2}{*}{$\begin{array}{c}\begin{array}{l}\text { Group N } \\
\text { no SC-1 }\end{array} \\
\text { All }\end{array}$} & \multirow{2}{*}{$\begin{array}{c}\begin{array}{c}\text { Group R } \\
\text { healthy }\end{array} \\
\text { Flow } \\
\text { cytometry }\end{array}$} \\
\hline & $\begin{array}{c}\text { Flow } \\
\text { cytometry }\end{array}$ & ELISA & & \\
\hline No. & 18 & 11 & 9 & 8 \\
\hline Male/Female & $9 / 9$ & $4 / 7$ & $8 / 1$ & $4 / 4$ \\
\hline \multicolumn{5}{|l|}{ Age } \\
\hline Median & 70 & 66 & 63 & 43 \\
\hline 1 st $/ 3$ rd quartile & $59 / 75$ & $58 / 72$ & $58 / 69$ & $30 / 57$ \\
\hline $\operatorname{Min} / \operatorname{Max}$ & $44 / 81$ & $44 / 79$ & $46 / 78$ & $24 / 61$ \\
\hline $\mathrm{P}$ to group $\mathrm{N}$ & 0.38 & 0.6 & & \\
\hline \multicolumn{5}{|l|}{$\begin{array}{l}\text { Tumor stages } \\
\text { (R0) }\end{array}$} \\
\hline Ia & 3 & 1 & 1 & \\
\hline $\mathrm{Ib}$ & 3 & 1 & 1 & \\
\hline II & 4 & 3 & 1 & \\
\hline IIIa & 1 & 0 & 2 & \\
\hline IIIb & 0 & 0 & 1 & \\
\hline IV & 3 & 3 & 2 & \\
\hline $\mathrm{R} 1 / 2$ & 4 & 3 & 1 & \\
\hline
\end{tabular}

Group S, flow cytometric (Flow Cyto.) and serum factor (ELISA) analysis were performed on different patients with the exception of 10 patients belonging to both analysis groups. Group N, patients were the same for both analyses. Tumor stages are according to UICC (15) for R0 resection.

Germany) and granulocyte-macrophage colony stimulating factor (GM-CSF EASIA, Biosource).

Flow cytometry. Blood was collected in EDTA containing tubes (S-Monovette, potassium-EDTA, Sarstedt) and $100 \mu 1$ of blood were stained with the following antibodies for $30 \mathrm{~min}$ at room temperature: CD45-FITC + CD14-PE (clone T29/33 + TÜK4; Dako, Hamburg, Germany), CD14-FITC (TÜK4; Dako), CD14-FITC + mouse IgG2a-PE isotype control (X39; BD Biosciences, Heidelberg, Germany), CD14-FITC + antiHLA-DR-PE (L243; BD), CD3-FITC + CD19-PE (SK7 + 4G7; BD), CD3-FITC + CD16-PE+CD56-PE, (SK7 + B73.1+MY31; BD), CD4-FITC (MT310; Dako), CD8-PE (DK25; Dako). The antibodies were used according to manufacturer's recommendation, at dilutions of 1:10 or 1:5. Red blood cells were lysed by addition of $2 \mathrm{ml}$ FACS Lysing Solution (BD). After $10 \mathrm{~min}$ incubation cells were pelleted (5 min, $450 \mathrm{~g}$, RT), washed with $1 \mathrm{ml}$ phosphate-buffered saline (PBS) and resuspended in $0.5 \mathrm{ml}$ PBS. Cells were measured with a FACScan flow cytometer (BD) set to 256 channel resolution and data analysis was performed with the program WinMDI. Lymphocytes were determined by the FSC/SSC properties of the cells, monocytes by CD14 staining. The HLA-DR expression on monocytes was calculated as the median fluorescence intensity (MFI) of HLA-DR-PE (in all channels) of all CD14+ monocytes. All 


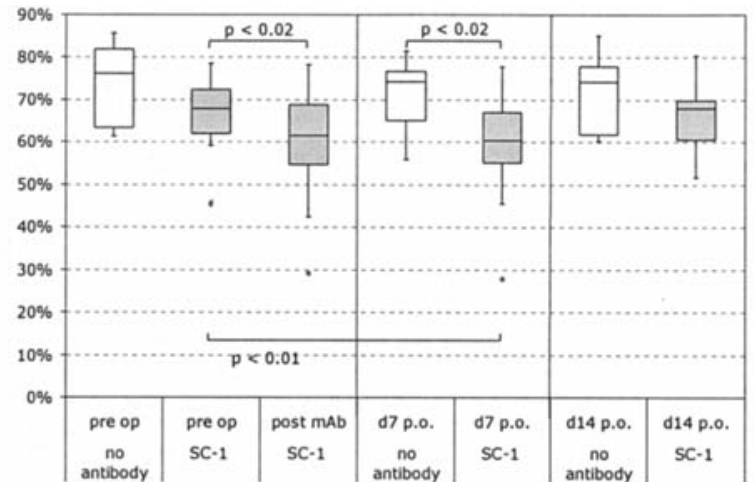

Figure 1. Percentage of $\mathrm{CD}^{+}{ }^{+} \mathrm{T}$-cells among lymphocytes was determined by flow cytometry and data are presented as box-and-whisker-plots. No antibody, patients without antibody treatment (group N); SC-1, patients treated with the SC-1 antibody (group S); pre-op, before surgery and antibody treatment; post $\mathrm{mAb}$, after antibody treatment and before surgery; $\mathrm{d} 7$ p.o., 7 days after surgery; d14 p.o., 14 days after surgery. Significant changes $(\mathrm{p}<0.02)$ are indicated.

other values are given as percentage of positive cells within the lymphocyte population.

Statistical analysis. For comparison of data within one group (e.g., before and after surgery) Wilcoxon matched-pairs signed-ranks test was used, between groups (group $\mathrm{S}$ vs. group N) Mann-Whitney U-Test. T and U values were calculated using Microsoft Excel Mac vX, probabilities were taken from standard statistical tables. Calculations were confirmed using R (16). Analysis of progression curves was performed according to Krauth (17): distribution tables were calculated with Excel Mac, probability of the table was determined with SAS for Unix (Release 8.2) using Fisher's exact test. Since a large number of tests were performed without any assumptions about the outcome, a low significance level of $\mathrm{p} \leq 0.01$ was chosen.

Values in the text are median values. Fig. 1 shows a boxand-whisker-plot according to Tukey (18). Upper and lower end of the box mark the 1st and 3rd quartile, the separating line the median. Whiskers indicate the minimum and maximum values unless they are further than $1.5 \mathrm{x}$ the interquartile range from the box borders. Values outside this range are indicated with a black dot as outliers.

\section{Results}

The immune status of patients undergoing the SC-1 antibody treatment was determined prior to antibody treatment (pre op), directly after antibody infusion but before surgery (post $\mathrm{mAb}), 7$ and 14 days after surgery (d7 p.o. and d14 p.o.). The immune status of the control patients was determined before surgery, 7 and 14 day after the operation. We wanted to address two questions: does the immune status change after antibody infusion and does the immune status of SC-1 treated patients differ from those without treatment. To address the first question, we compared the immune status before and after antibody application (Wilcoxon T-test). For the second question several comparisons summarized in Table II were performed. Additionally, a comparison of the time course according to Krauth (17) was performed.
Table II. Data comparison.

\begin{tabular}{|c|c|c|c|c|}
\hline \multicolumn{2}{|c|}{ Wilcoxon T-test } & \multicolumn{3}{|c|}{ Mann-Whitney U-test } \\
\hline Group & Comparison & Group 1 & Group 2 & Parameter \\
\hline S & $\begin{array}{l}\text { pre mAb vs. } \\
\text { post } m A b\end{array}$ & \multirow{3}{*}{ S } & \multirow{3}{*}{$\mathrm{N}$} & \multirow{3}{*}{$\begin{array}{l}\text { pre op } \\
\text { d7 p.o. } \\
\text { d14 p.o. } \\
\text { d7 p.o. - pre op } \\
\text { d14 p.o. - pre op }\end{array}$} \\
\hline $\begin{array}{l}S \\
N\end{array}$ & pre op vs. d7 p.o. & & & \\
\hline $\begin{array}{l}S \\
N\end{array}$ & pre op vs. d14 p.o. & & & \\
\hline \multirow[t]{2}{*}{$\mathrm{S}$} & \multirow[t]{2}{*}{ post mAb vs. d7 p.o. } & $S$ & $\mathrm{R}^{\mathrm{a}}$ & $\begin{array}{l}\text { pre op } \\
\text { d7 p.o. } \\
\text { d14 p.o. }\end{array}$ \\
\hline & & $\mathrm{N}$ & $\mathrm{R}^{\mathrm{a}}$ & $\begin{array}{l}\text { pre op } \\
\text { d7 p.o. } \\
\text { d14 p.o. }\end{array}$ \\
\hline
\end{tabular}

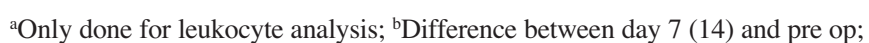
pre (post) mAb, before (after) antibody treatment; pre op, before surgery and antibody treatment; d7/14 p.o., 7 resp. 14 days after surgery.

Effects of the antibody treatment. After treatment with the monoclonal antibody SC-1 the values for IL6 were slightly increased but not to a statistically significant level (from 6.0 to $13.9 \mathrm{pg} / \mathrm{ml}$ (median), $\mathrm{p}=0.054)$. However, the serum level of the inflammatory cytokine TNF- $\alpha$ was clearly elevated: before antibody treatment, the median level was $26 \mathrm{pg} / \mathrm{ml}$, after antibody treatment $36 \mathrm{pg} / \mathrm{ml}(\mathrm{p}=0.004)$ (Table III). The TNF- $\alpha$ serum level remained elevated after the operation (d7 p.o., $35 \mathrm{pg} / \mathrm{ml}$; d14 p.o., $35 \mathrm{pg} / \mathrm{ml}$ ). Although this increase seems to be significant, it is probably of no clinical relevance, since values up to $39 \mathrm{pg} / \mathrm{ml} \mathrm{TNF-} \alpha$ can be found in healthy individuals (Table VI). Considering that the patients underwent a massive surgical procedure the TNF- $\alpha$ values measured after operation can only be considered as optimal.

After antibody treatment the fraction of lymphocytes was slightly reduced from 14.8 to $13.8 \%$, ( $\mathrm{p}=0.027$, Table IV) as well as the portion of T-cells $\left(\mathrm{CD}^{+}\right)$among the lymphocytes from 67.8 to $61.5 \%$ ( $\mathrm{p}=0.018$ ) (Fig. 1 ).

Again these changes are only minimal and the values obtained after antibody treatment are still in the range of healthy control individuals (group R) (Table V). The reduction of T-cells seems to be caused mainly by $\mathrm{CD}^{+}$helper T-cells, since their values drop from 45.4 to $42.7 \%(\mathrm{p}=0.14$ ) while the $\mathrm{CD}^{+}$T-cells slightly increase from 22.5 to $23.9 \%(\mathrm{p}=0.77)$.

Overall, the infusion of the antibody did not cause any clinically significant change in the immune status of the patients.

Changes of immune status after surgery. Seven days after surgery the fraction of lymphocytes (14.8 vs. $8.1 \%, \mathrm{p}=0.003)$, $\mathrm{CD}^{+}$T-cells $(67.8$ vs. $60.4 \%, \mathrm{p}=0.009$, Fig. 1$), \mathrm{CD}^{+}$cytotoxic T-cells (22.5 vs. $17.0 \%, \mathrm{p}=0.003)$ and the HLA-DR expression on monocytes (MFI: 821 vs. 422, p=0.001) were 
Table III. Serum factor analysis.

\begin{tabular}{|c|c|c|c|c|c|c|c|c|c|c|c|c|}
\hline & \multicolumn{2}{|c|}{$\mathrm{TNF}-\alpha(\mathrm{pg} / \mathrm{ml})$} & \multicolumn{2}{|c|}{ IL12p70 (pg/ml) } & \multicolumn{2}{|c|}{ IL6 (pg/ml) } & \multicolumn{2}{|c|}{$\mathrm{IFN}-\gamma(\mathrm{IU} / \mathrm{ml})$} & \multicolumn{2}{|c|}{ Neopterin $(\mathrm{nM})$} & \multicolumn{2}{|c|}{ GM-CSF $(\mathrm{pg} / \mathrm{ml})$} \\
\hline & Group S & Group N & Group S & Group N & Group S & Group N & Group S & Group N & Group S & Group N & Group S & Group N \\
\hline \multicolumn{13}{|l|}{ pre-op } \\
\hline Median & 26.4 & 27.3 & 17.3 & 21.8 & 6 & 5.5 & 0.3 & 1.3 & 6.6 & 3.3 & 62 & 40.4 \\
\hline $1 \mathrm{st} / 3 \mathrm{rd}$ quartile & $23.4 / 29.5$ & $25.8 / 29.7$ & $9.4 / 33.4$ & $8.24 / 22.5$ & $4 / 11$ & $3.4 / 8.8$ & $0.1 / 0.5$ & $1.1 / 1.4$ & $5.1 / 7.5$ & $2.7 / 4.3$ & $45.7 / 66.3$ & $36.4 / 43.5$ \\
\hline $\operatorname{Min} / \operatorname{Max}$ & $22.1 / 37.2$ & $20.8 / 40$ & $5.9 / 80.8$ & $4.91 / 30.1$ & $2.4 / 701$ & $3.1 / 64.2$ & $0 / 0.7$ & $0.8 / 2.1$ & $2.6 / 12$ & $2.6 / 7.8$ & $26.4 / 150$ & $33.8 / 190$ \\
\hline $\mathrm{p}$ to group $\mathrm{N}$ & 0.47 & & 0.761 & & 0.79 & & 0.0002 & & 0.08 & & 0.254 & \\
\hline \multicolumn{13}{|l|}{ post mAb } \\
\hline Median & 36 & & 19.3 & & 13.9 & & 0.4 & & 6.5 & & 54.9 & \\
\hline $1 \mathrm{st} / 3$ rd quartile & $33 / 43.8$ & & $10.6 / 46.7$ & & $7.7 / 37.8$ & & $0 / 0.8$ & & $5.2 / 10.1$ & & $42.9 / 63.4$ & \\
\hline $\operatorname{Min} / \operatorname{Max}$ & $24.1 / 56.5$ & & $4.3 / 92.8$ & & $2.3 / 725$ & & $0 / 1$ & & $1.7 / 13.8$ & & $34.7 / 150$ & \\
\hline $\begin{array}{l}\mathrm{p} \text { to group N/ } \\
\text { pre-op }\end{array}$ & $-/ 0.004$ & & $-/ 0.83$ & & $-/ 0.054$ & & $-/ 0.407$ & & $-/ 0.41$ & & $-/ 0.966$ & \\
\hline \multicolumn{13}{|l|}{ d7 p.o. } \\
\hline Median & 35 & 27.9 & 25 & 17.7 & 56.1 & 49.8 & 0.5 & 1 & 7 & 3.5 & 58.9 & 42.2 \\
\hline $1 \mathrm{st} / 3$ rd quartile & $30.6 / 40.8$ & $27.4 / 42$ & $11.1 / 42.5$ & $10.3 / 19.1$ & $24.7 / 96.5$ & $24.2 / 112$ & $0.4 / 0.7$ & $1 / 1.1$ & $3.9 / 9.2$ & $2.7 / 8.9$ & $56.9 / 76.3$ & $33.6 / 59.5$ \\
\hline $\operatorname{Min} / \operatorname{Max}$ & $19.5 / 86.8$ & $25.1 / 45.7$ & $2.6 / 61.7$ & $4.5 / 25.5$ & $11.1 / 452$ & $12.9 / 413$ & $0 / 0.9$ & $0.8 / 1.6$ & $2.8 / 13.2$ & $2.3 / 15.8$ & $34.9 / 118$ & $26.4 / 101$ \\
\hline $\begin{array}{l}\mathrm{p} \text { to group } \mathrm{N} / \\
\text { pre-op }\end{array}$ & $0.38 / \mathbf{0 . 0 0 5}$ & $-/ 0.129$ & $0.18 / 1$ & $-/ 0.192$ & $0.94 / 0.054$ & $-/ 0.039$ & $0.0004 / 0.126$ & $-/ 0.066$ & $0.3 / 0.92$ & $-/ 0.1$ & $0.046 / 0.64$ & $-/ 0.359$ \\
\hline \multicolumn{13}{|l|}{ d14 p.o. } \\
\hline Median & 35 & 41.1 & 23.1 & 11.4 & 50.9 & 16.4 & 0.5 & 1.3 & 5.2 & 4.6 & 68.8 & 44.5 \\
\hline 1st/3rd quartile & $25.8 / 44.8$ & $36.8 / 49.3$ & $19.4 / 36.1$ & $9 / 16.3$ & $22.9 / 72.1$ & $10.1 / 136$ & $0.3 / 0.8$ & $0.9 / 1.4$ & $4.4 / 7.9$ & $3.6 / 5.2$ & $62.8 / 86.1$ & $37.3 / 58.9$ \\
\hline Min/Max & $18.9 / 87.8$ & $26.6 / 62.3$ & $6.5 / 58.1$ & $3.5 / 21.8$ & $7.1 / 455$ & $7.6 / 2613$ & $0 / 1.1$ & $0.8 / 2$ & $2.5 / 19.5$ & $3.1 / 20.8$ & $34.5 / 105.2$ & $33.2 / 92.3$ \\
\hline $\begin{array}{l}\mathrm{p} \text { to group N/ } \\
\text { pre-op }\end{array}$ & $0.23 / 0.032$ & $-/ 0.004$ & $\mathbf{0 . 0 1} / 0.64$ & $-/ 0.098$ & $0.77 / 0.054$ & $-/ 0.055$ & $0.001 / 0.07$ & $-/ 1$ & $0.68 / 0.7$ & $-/ 0.14$ & $0.056 / 0.48$ & $-/ 1$ \\
\hline
\end{tabular}

For each factor and time-point, the median values (1st line), the 1 st and 3 rd quartile (2nd line) and the minimum and maximum (3rd line) values are shown. In the left column of the last line, the p-values in comparison to group $\mathrm{N}$ and in the right column the p-values in comparison to the pre-operation values are shown. $\mathrm{P}$-values $\leq 0.01$ are in bold (except for IFN- $\gamma$, differences are due to technical reasons). For abbreviations see legend of Table II.

clearly reduced (Table IV). Probably as a consequence the percentage of CD19+ B-cells increased (12.4 vs. 17.1\%, $\mathrm{p}=0.002)$. Similarly, the CD4/CD8 ratio was increased since the number of $\mathrm{CD}^{+}$helper T-cells did not change. However, similar changes could be observed in patients without the $\mathrm{SC}-1$ treatment (group N). Comparing the values from the SC-1 treated (group S) and untreated patients before surgery and on day 7 after surgery no significant difference could be observed except for the fraction of $\mathrm{CD}^{+} \mathrm{T}$-cells on day 7 after surgery (group S, 60.4\%; group N, 74.1\%; $\mathrm{p}=0.011$, Fig. 1). On the other hand, the percentage of $\mathrm{CD}^{+} \mathrm{T}$-cells was already lower in the group $\mathrm{S}$ before the surgery (67.8 vs. $76.2 \%, \mathrm{p}=0.06$ ), although this difference was not statistically significant. Comparing the change of $\mathrm{CD}^{+} \mathrm{T}$-cells (percentage at day $7 /$ percentage before surgery) between $\mathrm{SC}-1$ treated and untreated patients, again no statistically significant change could be observed (group S, $-6.9 \%$; group N, $-4.3 \%$; $\mathrm{p}=0.258$ ).

Thus, all the changes observed were due to the surgical treatment and not due to the SC-1 treatment. The observed changes after surgery are common and have been repeatedly described in the literature (19-23).
Two weeks (14 days) after surgery the values for $\mathrm{CD} 19^{+}$ $\mathrm{B}$-cells, $\mathrm{CD}^{+}$and $\mathrm{CD}^{+}$cytotoxic T-cells returned to the normal values before surgery, while the values for lymphocytes and HLA-DR expression on monocytes stayed reduced (lymphocytes, $8.8 \%, \mathrm{p}=0.001$ to pre op; HLA-DR, MFI 524, $\mathrm{p}=0.008)$. Again the same pattern could be observed in patients without antibody treatment showing no significant difference to the $\mathrm{SC}-1$ treated patients (lymphocytes: group $\mathrm{S}$, $8.8 \%$; group N, 9.0\%, $\mathrm{p}=0.44$; HLA-DR, group S, MFI 524; group $\mathrm{N}, \mathrm{MFI} 750, \mathrm{p}=0.28$ )

TNF- $\alpha$ levels remained on the slightly elevated levels obtained after antibody treatment and did not return to lower levels in the time period observed (Table III). No other serum factors changed significantly.

Comparing the SC-1 treated group with the untreated group. We compared the median values of all parameters before treatment, on day 7 and day 14 after surgery. In nearly all cases, the differences between the two groups were not statistically relevant $(\mathrm{p}>0.1)$. Only 14 days post-surgery the IL12p70 values were statistically higher in group $S$ than in 
Table IV. Flow cytometric analysis.

\begin{tabular}{|c|c|c|c|c|c|c|c|c|c|c|}
\hline & \multicolumn{2}{|c|}{ Lymphocytes } & \multicolumn{2}{|c|}{ Monocytes } & \multicolumn{2}{|c|}{$\mathrm{CD}^{+}$lymphocytes } & \multicolumn{2}{|c|}{ CD4+ lymphocytes } & \multicolumn{2}{|c|}{ CD8 $8^{+}$lymphocytes } \\
\hline & Group S & Group N & Group S & Group N & Group S & Group N & Group S & Group N & Group S & Group $\mathrm{N}$ \\
\hline \multicolumn{11}{|l|}{ pre-op } \\
\hline Median & 14.8 & 20.3 & 5.5 & 4.4 & 67.8 & 76.2 & 45.4 & 47.3 & $22.5^{\mathrm{a}}$ & 28.0 \\
\hline 1st/3rd quartile & $11.3 / 20.6$ & $18.4 / 22.6$ & $4.4 / 7.2$ & $3.3 / 6.8$ & $61.9 / 72.3$ & $63.3 / 82.0$ & $37.8 / 50.9$ & $36.6 / 52.0$ & $13.4 / 28.1$ & $17.7 / 34.6$ \\
\hline $\operatorname{Min} / \operatorname{Max}$ & $6.8 / 37.3$ & $6.5 / 33.1$ & $1.5 / 10.4$ & $1.8 / 10.3$ & $45.2 / 78.5$ & $61.3 / 85.6$ & $27.1 / 61.5$ & $30.7 / 61.9$ & $8.7 / 35.8$ & $8.4 / 39.6$ \\
\hline $\mathrm{p}$ to group N/pre-op & $0.212 /-$ & & $0.275 /-$ & & $0.060 /-$ & & $0.743 /-$ & & $0.275 /-$ & \\
\hline \multicolumn{11}{|l|}{ post $\mathrm{mAb}$} \\
\hline Median & $13.8^{\mathrm{a}}$ & & 4.9 & & 61.5 & & 42.7 & & 23.9 & \\
\hline 1st/3rd quartile & $8.8 / 18.4$ & & $3.1 / 7.7$ & & $54.7 / 68.7$ & & $30.9 / 48.0$ & & $12.3 / 29.4$ & \\
\hline $\operatorname{Min} / \operatorname{Max}$ & $6.9 / 34.6$ & & $2.8 / 9.2$ & & $29.1 / 78.3$ & & $19.7 / 55.3$ & & $6.0 / 40.4$ & \\
\hline p to group N/pre-op & $-/ 0.027$ & & $-/ 0.523$ & & $-/ 0.018$ & & $-/ 0.142$ & & $-/ 0.766$ & \\
\hline \multicolumn{11}{|l|}{ d7 p.o. } \\
\hline Median & $8.1^{\mathrm{a}}$ & $8.5^{\mathrm{a}}$ & 5.8 & 6.8 & 60.4 & 74.1 & 43.9 & 47.3 & 17.0 & 26.3 \\
\hline 1st/3rd quartile & $6.9 / 9.1$ & $8.3 / 9.8$ & $4.2 / 7.9$ & $5.0 / 8.2$ & $55.0 / 67.0$ & $65.0 / 76.7$ & $37.8 / 50.2$ & $38.9 / 48.2$ & $11.3 / 19.5$ & $13.5 / 30.4$ \\
\hline Min/Max & $5.0 / 18.0$ & $3.2 / 15.5$ & $3.3 / 11.3$ & $3.7 / 14.3$ & $27.8 / 77.7$ & $55.9 / 81.4$ & $12.6 / 53.8$ & $33.3 / 64.6$ & $6.7 / 34.7$ & $6.6 / 37.6$ \\
\hline $\mathrm{p}$ to group $\mathrm{N} / \mathrm{pre-op}$ & $0.67 / \mathbf{0 . 0 0 3}$ & $-/ 0.008$ & $0.43 / 0.67$ & $-/ 0.16$ & $0.01 / \mathbf{0 . 0 0 9}$ & $-/ 0.359$ & $0.49 / 0.3$ & $-/ 0.5$ & $0.11 / \mathbf{0 . 0 0 3}$ & $-/ 0.074$ \\
\hline \multicolumn{11}{|l|}{ d14 p.o } \\
\hline Median & $8.8^{\mathrm{a}}$ & $9.0^{\mathrm{a}}$ & 5.0 & 4.4 & 67.9 & 74.1 & 43.2 & 51.0 & $19.1^{\mathrm{a}}$ & $27.7^{\mathrm{a}}$ \\
\hline 1 st/3rd quartile & $5.4 / 10.7$ & $6.9 / 13.1$ & $4.1 / 6.1$ & $3.0 / 5.4$ & $60.5 / 69.8$ & $61.8 / 77.9$ & $38.0 / 49.7$ & $35.5 / 51.9$ & $13.0 / 25.5$ & $13.9 / 28.3$ \\
\hline Min/Max & $3.0 / 18.0$ & $5.7 / 22.1$ & $1.6 / 8.8$ & $1.7 / 13.0$ & $51.7 / 80.4$ & $60.2 / 85.0$ & $24.3 / 57.2$ & $30.5 / 66.1$ & $6.6 / 35.3$ & $6.7 / 34.2$ \\
\hline \multirow[t]{2}{*}{$\mathrm{p}$ to group N/pre-op } & $0.44 / \mathbf{0 . 0 0 1}$ & $-/ 0.008$ & $0.40 / 0.47$ & $-/ 0.16$ & $0.13 / 0.58$ & $-/ 0.359$ & $0.3 / 0.67$ & 0.5 & $0.375 / 0.12$ & $-/ 0.074$ \\
\hline & \multicolumn{2}{|c|}{ CD4/CD8 } & \multicolumn{2}{|c|}{ CD19+ lymphocytes } & \multicolumn{2}{|c|}{ CD $16^{+} / 56^{+}$lymphocytes } & \multicolumn{2}{|c|}{ HLA-DR monocytes } & & \\
\hline \multicolumn{11}{|l|}{ pre-op } \\
\hline Median & 2.06 & 1.37 & 12.4 & 8.0 & 13.4 & 12.8 & 821 & 931 & & \\
\hline 1st/3rd quartile & $1.42 / 3.17$ & $1.18 / 2.89$ & $10.0 / 14.3$ & 7.3/9.6 & $8.9 / 17.9$ & $7.3 / 16.1$ & $480 / 1027$ & $805 / 1286$ & & \\
\hline Min/Max & $0.76 / 5.51$ & $0.84 / 6.19$ & $2.7 / 19.1$ & $6.1 / 14.7$ & $2.9 / 38.7$ & $4.4 / 21.9$ & $229 / 1486$ & $698 / 1715$ & & \\
\hline $\mathrm{p}$ to group $\mathrm{N} / \mathrm{pre}-\mathrm{op}$ & $0.596 /-$ & & $0.095 /-$ & & $0.9 /-$ & & $0.19 /-$ & & & \\
\hline \multicolumn{11}{|l|}{ post $\mathrm{mAb}$} \\
\hline Median & 1.75 & & 11.0 & & 14.7 & & 898 & & & \\
\hline 1st/3rd quartile & $1.45 / 3.61$ & & $8.4 / 13.1$ & & $9.3 / 22.5$ & & $511 / 1065$ & & & \\
\hline Min/Max & $0.61 / 7.29$ & & $1.6 / 20.5$ & & $3.4 / 42.2$ & & $274 / 3106$ & & & \\
\hline $\mathrm{p}$ to group $\mathrm{N} /$ pre-op & $-/ 0.832$ & & $-/ 0.06$ & & $-/ 0.154$ & & $-/ 0.167$ & & & \\
\hline \multicolumn{11}{|l|}{ d7 p.o. } \\
\hline Median & $2.57^{\mathrm{a}}$ & $1.58^{\mathrm{a}}$ & 17.1 & 11.0 & 11.7 & 12.5 & $422^{\mathrm{a}}$ & 316 & & \\
\hline 1st/3rd quartile & $1.63 / 3.81$ & $1.45 / 3.72$ & $11.7 / 21.9$ & $8.2 / 14.4$ & $8.2 / 14.5$ & 7.7/13.6 & $305 / 487$ & $294 / 604$ & & \\
\hline Min/Max & $1.27 / 7.68$ & $1.04 / 9.54$ & $1.6 / 31.7$ & $6.8 / 18.7$ & $1.5 / 20.9$ & $2.9 / 16.9$ & $90 / 1000$ & $143 / 698$ & & \\
\hline $\mathrm{p}$ to group N/pre-op & $0.53 / \mathbf{0 . 0 0 4}$ & $-/ 0.055$ & $0.06 / \mathbf{0 . 0 0 2}$ & $-/ 0.098$ & $0.86 / 0.11$ & $-/ 0.155$ & $0.83 / \mathbf{0 . 0 0 1}$ & $-/ 0.004$ & & \\
\hline \multicolumn{11}{|l|}{ d14 p.o } \\
\hline Median & $2.04^{\mathrm{a}}$ & 1.81 & 14.1 & 8.9 & 11.4 & 11.1 & 524 & 750 & & \\
\hline 1st/3rd quartile & $1.71 / 3.42$ & $1.62 / 4.65$ & $10.4 / 17.1$ & 7.0/10.2 & $8.1 / 18.0$ & $6.6 / 17.5$ & $327 / 707$ & $407 / 931$ & & \\
\hline Min/Max & $0.89 / 8.46$ & $1.04 / 7.75$ & $2.9 / 24.3$ & $4.6 / 14.2$ & $3.2 / 27.0$ & $3.2 / 21.0$ & $32 / 1596$ & $120 / 1382$ & & \\
\hline $\mathrm{p}$ to group N/pre-op & $1.0 / 0.231$ & $-/ 0.055$ & $0.01 / 0.024$ & $-/ 0.098$ & $0.98 / 0.3$ & $-/ 0.155$ & $0.28 / \mathbf{0 . 0 0 8}$ & $-/ 0.004$ & & \\
\hline
\end{tabular}

Lymphocytes and monocytes values are percentage of total leukocytes. $\mathrm{CD} 3^{+}, \mathrm{CD}^{+}, \mathrm{CD} 8^{+}, \mathrm{CD} 19^{+}, \mathrm{CD} 16 / 56^{+}$values are percentages of lymphocytes. HLA-DR on mono-

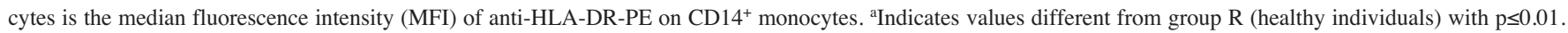
Further explanations in legends to Tables II and III.

group N (group S, $23.1 \mathrm{pg} / \mathrm{ml}$; group $\mathrm{N}, 11.4 \mathrm{pg} / \mathrm{ml}, \mathrm{p}=0.010$ ). However, previous values for group $\mathrm{S}$ were in the same range
(Table III) and did not change significantly after antibody treatment or surgery. It seems that this difference is more due 
Table V. Flow cytometric data from group R (healthy individuals).

\begin{tabular}{|c|c|c|c|c|c|}
\hline & \multicolumn{5}{|c|}{ Reference values } \\
\hline & Lymphocytes (\%) & Monocytes (\%) & CD3 (\%) & CD4 (\%) & $\mathrm{CD} 8(\%)$ \\
\hline Median & 21.7 & 6.9 & 68.5 & 38.6 & 33.4 \\
\hline 1 st/3rd quartile & $20.7 / 26.5$ & $6.1 / 7.3$ & $66.5 / 70.8$ & $33.6 / 41.7$ & $30.3 / 35.6$ \\
\hline Min/Max & $17.1 / 29.6$ & $5.1 / 8.1$ & $63.3 / 78.6$ & $32.4 / 63.0$ & $20.6 / 39.8$ \\
\hline \multirow[t]{2}{*}{$95 \%$ range $\mathrm{BD}$} & & & $59.0-85.0$ & $29.0-61.0$ & $11.0-38.0$ \\
\hline & $\mathrm{CD} 4 / \mathrm{CD} 8$ & CD19 (\%) & CD16+56 (\%) & \multicolumn{2}{|c|}{ MFI of HLA-DR monocytes } \\
\hline Median & 1.16 & 9.3 & 13.8 & \multicolumn{2}{|c|}{692} \\
\hline 1 st $/ 3$ rd quartile & $0.93 / 1.42$ & $7.8 / 10.7$ & $9.3 / 16.6$ & \multicolumn{2}{|c|}{$571 / 1019$} \\
\hline Min/Max & $0.81 / 3.06$ & $2.7 / 13.0$ & $5.2 / 24.6$ & \multicolumn{2}{|c|}{$264 / 1201$} \\
\hline $95 \%$ range $\mathrm{BD}$ & $0.9-3.6$ & $6.4-23.0$ & $5.6-31.0$ & & \\
\hline
\end{tabular}

Median. 1st and 3rd quartile. Minimum and maximum value obtained from healthy volunteers (Group R). Bottom line, 95\% range from product information of BD Biosciences. MFI, median fluorescence intensity.

Table VI. Properties of ELISA assays.

\begin{tabular}{|c|c|c|c|}
\hline & TNF- $\alpha(\mathrm{pg} / \mathrm{ml})$ & IL12p70 (pg/ml) & IL6 (pg/ml) \\
\hline Sensitivity & 3.0 & 0.5 & 2 \\
\hline \multicolumn{4}{|l|}{ Reference range } \\
\hline ELISA manual & $0-20$ & $0-4.5$ & $0-8.5$ \\
\hline BD Biosciences & $0-21$ & $0-4.0$ & $0-12.1$ \\
\hline Milenia (DPC) & $0-39$ & & $0-28.0$ \\
\hline \multirow[t]{2}{*}{ Range of standards } & $14-1430$ & $1.56-100$ & $16-1690$ \\
\hline & $\begin{array}{l}\text { IFN- } \gamma \\
(\mathrm{IU} / \mathrm{ml})\end{array}$ & $\begin{array}{l}\text { Neopterin } \\
\quad(\mathrm{nM})\end{array}$ & $\begin{array}{l}\text { GM-CSF } \\
(\mathrm{pg} / \mathrm{ml})\end{array}$ \\
\hline Sensitivity & 0.03 & 2 & 3 \\
\hline \multicolumn{4}{|l|}{ Reference range } \\
\hline ELISA manual & $0-1.2$ & $3.0-10.0$ & $0-14.0$ \\
\hline BD Biosciences & $0-1.8$ & & $0-1.8$ \\
\hline \multicolumn{4}{|l|}{ Milenia (DPC) } \\
\hline Range of standards & $0.85-28$ & $2-250$ & $50-2000$ \\
\hline \multicolumn{4}{|c|}{$\begin{array}{l}\text { Sensitivity as indicated in the product information. Reference range for } \\
\text { healthy individuals: 'ELISA manual' reference range as indicated in the } \\
\text { product information of the assay actually used. Other data are reference } \\
\text { ranges from product information of the indicated manufacturer. Range of } \\
\text { standards indicates the lowest }(>0) \text { and highest standard used. }\end{array}$} \\
\hline
\end{tabular}

to an extremely low value in group $\mathrm{N}$, which is lower than the values before surgery (pre op) and 7 days after surgery (d7 p.o.) in this group (although the change is not statistically relevant).
Comparison to healthy individuals. Since the number of lymphocytes and $\mathrm{CD} 8^{+} \mathrm{T}$-cells dropped after surgery, these numbers were significantly different from the values obtained from healthy volunteers (group R) (Tables IV and V). Due to the reduced $\mathrm{CD}^{+} \mathrm{T}$-cell values, the $\mathrm{CD} 4 / \mathrm{CD} 8$ ratio was increased compared to group $\mathrm{R}$.

However, all these values were still within published ranges for healthy individuals (Table V). Thus, although values were reduced, they were still within a normal range and did not indicate any pathological state.

Similarly, the concentration of serum factors before surgery was within the range of healthy individuals, except for elevated levels of IL12p70 and GM-CSF. After surgery, the values of IL6 increased over normal levels due to stress induced by the operation. In group N the IL6 values returned to normal levels 14 days after surgery while in group $\mathrm{S}$ the values stayed above normal values. The values for IL12p70 and GM-CSF were higher than normal after antibody treatment and after surgery, but did not change compared to the values before operation. The IFN- $\gamma$ values were extremely low in both groups at all time-points measured. The slightly higher values in group $\mathrm{N}$ result from the measurement of these values on a different ELISA plate (all values were lower or around the value of the lowest standard).

Progression curve. Since all previous analyses are based on pairwise comparisons, we tried to analyze the data using an approach incorporating all values measured (pre op and pre SC-1), day 7 and day 14 after surgery. The values obtained after antibody treatment were omitted since there was no comparable value in the group $\mathrm{N}$. We chose the T1 evaluation according to Krauth (17). None of the parameters tested showed a significant difference in the progression curve between the SC-1 treated group S and the untreated group N (Table VII). 
Table VII. Progression analysis.

\begin{tabular}{|c|c|c|c|c|c|c|c|c|c|c|c|c|}
\hline & \multicolumn{2}{|c|}{ TNF- $\alpha(\mathrm{pg} / \mathrm{ml})$} & \multicolumn{2}{|c|}{ IL12p70 (pg/ml) } & \multicolumn{2}{|c|}{ IL6 (pg/ml) } & \multicolumn{2}{|c|}{$\mathrm{IFN}-\gamma(\mathrm{IU} / \mathrm{ml})$} & \multicolumn{2}{|c|}{ Neopterin (nM) } & \multicolumn{2}{|c|}{ GM-CSF (pg/ml) } \\
\hline & $\mathrm{SC}-1$ & Control & SC-1 & Control & SC-1 & Contro & SC-1 & Control & SC-1 & Control & SC-1 & Control \\
\hline \multicolumn{13}{|l|}{ Difference: } \\
\hline \multicolumn{13}{|l|}{ d7 p.o.-pre-op } \\
\hline Median & 10 & 2 & -1.9 & -2.7 & 18.9 & 32.9 & 0.1 & -0.2 & 1 & 0.8 & 1 & -4.3 \\
\hline 1st/3rd quartile & 7/11.7 & $0.3 / 7.1$ & $-11.3 / 20.2$ & $-4.3 / 1$ & $14 / 55.4$ & $13.4 / 10$ & $-0.1 / 0$ & $-0.3 / 0$ & $-1.7 / 2.1$ & $-0.1 / 2.1$ & $-7.5 / 16.3$ & $-6.6 /-1.3$ \\
\hline Min/Max & $-3.3 / 57.5$ & $-2.5 / 18.6$ & $-51 / 39.2$ & $-12.4 / 3.7$ & $-249 / 11$ & $-40 / 40$ & $-0.2 / 0$ & $0.9-0.7 / 0.2$ & $-7.9 / 7$ & $-0.6 / 8$ & $-38.8 / 51.4$ & $-89 / 25.5$ \\
\hline $\mathrm{p}$ to group $\mathrm{N}$ & 0.16 & 0.595 & 0.71 & 0.012 & 0.71 & 0.331 & & & & & & \\
\hline \multicolumn{13}{|l|}{ Difference: } \\
\hline \multicolumn{13}{|l|}{ d14 p.o.-pre-op } \\
\hline Median & 7.5 & 11.5 & 6.7 & -1.7 & 20.6 & 7.6 & 0.2 & 0 & -1.8 & 0.9 & 11.8 & 2.3 \\
\hline 1 st/3rd quartile & $-1.2 / 13.7$ & $9.8 / 13.5$ & $-11.9 / 10.3$ & $-11.1 /-0$. & $4.2 / 50$ & $5.3 / 12$ & $0 / 0.5$ & $-0.2 / 0.2$ & $-2.3 / 1.9$ & $0.3 / 2.5$ & $-6.4 / 29.1$ & $-6.5 / 15.4$ \\
\hline Min/Max & $-4.1 / 58.5$ & $1.2 / 35.2$ & $-47.9 / 37$ & $-21.1 / 8.1$ & $-246 / 70$ & $.3-54.1 / 26$ & $-0.2 / 0$ & $\begin{array}{cc}0.9 & -0.4 / 0.3\end{array}$ & $-4.7 / 7.7$ & $-2.9 / 13$ & $-70.5 / 67.9$ & $-98 / 52$ \\
\hline $\mathrm{p}$ to group $\mathrm{N}$ & 0.425 & & 0.27 & & 0.766 & & 0.14 & & 0.175 & & 0.603 & \\
\hline \multirow[t]{2}{*}{ Progression curve } & $\mathrm{p}=0.063$ & & $\mathrm{p}=0.488$ & & $\mathrm{p}=0.59$ & & $\mathrm{p}=0.2$ & & $\mathrm{p}=0.450$ & & $\mathrm{p}=0.450$ & \\
\hline & \multicolumn{2}{|c|}{ Lymphocytes } & \multicolumn{2}{|c|}{ Monocytes } & \multicolumn{2}{|c|}{$\mathrm{CD}^{+}$lymphocytes } & \multicolumn{2}{|c|}{$\mathrm{CD}^{+}$lymphocytes } & \multicolumn{2}{|c|}{$\mathrm{CD} 8^{+}$lymhocytes } & \multicolumn{2}{|c|}{$\mathrm{CD} 4 / \mathrm{CD} 8$} \\
\hline \multicolumn{13}{|l|}{ Difference: } \\
\hline \multicolumn{13}{|l|}{ d7 p.o.-pre-op } \\
\hline Median & -6.8 & -9.9 & 0.4 & 0.6 & -6.9 & -4.3 & -2.5 & 2.7 & -3.8 & -2.5 & 0.54 & 0.32 \\
\hline 1st/3rd quartile & $-11.7 /-3.8$ & $-15.1 /-5.2$ & $-1.6 / 2.3$ & $-0.4 / 4.3$ & $-12.0 /-1.5$ & $-5.5 / 0.3$ & $-7.4 / 2.4$ & $-3.3 / 5.8$ & $-8.1 /-1.5$ & $-4.3 /-1.8$ & $0.16 / 0.73$ & $0.20 / 0.83$ \\
\hline Min/Max & $-28.3 / 11.2$ & $-23.4 / 1.9$ & $-5.5 / 6.9$ & $-1.8 / 12.5$ & $-33.1 / 29.1$ & $-18.4 / 10.8$ & $-22.1 / 23.9$ & $-8.5 / 10.7$ & $-17.5 / 6.5$ & $-10.1 / 4.8$ & $-0.75 / 73.14$ & $-0.62 / 3.35$ \\
\hline $\mathrm{p}$ to group $\mathrm{N}$ & 0.375 & & 0.463 & & 0.258 & & 0.212 & & 0.561 & & 0.900 & \\
\hline \multicolumn{13}{|l|}{$\begin{array}{l}\text { Difference: } \\
\text { d14 p.o.-pre-op }\end{array}$} \\
\hline Median & -6.6 & -11.0 & -0.3 & -1.5 & -1.2 & -1.7 & 0.2 & 0.2 & -4.2 & -4.2 & 0.39 & 0.50 \\
\hline 1st/3rd quartile & $-12.0 /-2.1$ & $-14.0 /-7.9$ & $-2.1 / 0.9$ & $-2.3 / 1.0$ & $-6.4 / 2.1$ & $-4.1 /-0.6$ & $-5.9 / 4.3$ & $-0.7 / 3.4$ & $-5.1 /-0.2$ & $-5.8 /-1.7$ & $-0.42 / 0.94$ & $0.20 / 1.26$ \\
\hline $\operatorname{Min} / \operatorname{Max}$ & $-26.9 / 4.6$ & $-14.6 / 4.8$ & $-4.3 / 4.4$ & $-4.9 / 11.2$ & $-16.4 / 24.6$ & $-13.5 / 1.6$ & $-22.2 / 19.2$ & $-5.5 / 7.2$ & $-11.0 / 9.0$ & $-12.6 / 2.6$ & $-2.10 / 2.94$ & $-0.39 / 1.76$ \\
\hline $\mathrm{p}$ to group $\mathrm{N}$ & 0.463 & & 0.860 & & 0.495 & & 0.643 & & 0.528 & & 0.554 & \\
\hline \multirow[t]{2}{*}{ Progression curve } & $\mathrm{p}=0.254$ & & $\mathrm{p}=0.331$ & & $\mathrm{p}=0.475$ & & $\mathrm{p}=0.712$ & & $\mathrm{p}=0.587$ & & $\mathrm{p}=0.313$ & \\
\hline & \multicolumn{2}{|c|}{$\begin{array}{c}\mathrm{CD} 19^{+} \\
\text {lymphocytes }\end{array}$} & $\begin{array}{l}\mathrm{CD} 16^{+} \\
\text {lymphoc }\end{array}$ & $\begin{array}{l}+/ 56^{+} \\
\text {cytes }\end{array}$ & $\begin{array}{l}\text { HLA- } \\
\text { monoc }\end{array}$ & $\begin{array}{l}\text {-DR } \\
\text { cytes }\end{array}$ & & & & & & \\
\hline Difference: & & & & & & & & & & & & \\
\hline d7 p.o.-pre-op & & & & & & & & & & & & \\
\hline Median & 4.2 & 2.1 & -1.8 & -2.2 & -433 & 636 & & & & & & \\
\hline 1st/3rd quartile & $2.4 / 6.3$ & $0.3 / 3.7$ & $-6.4 / 1.9$ & $-4.7 / 0.4$ & $-684 /-216$ & $-829 /-508$ & & & & & & \\
\hline Min/Max & $-6.9 / 17.2$ & $-6.8 / 10.8$ & $-23.5 / 7.3$ & $-6.9 / 3.2$ & $-1093 / 295$ & $-1018 /-201$ & & & & & & \\
\hline $\mathrm{p}$ to group $\mathrm{N}$ & 0.275 & & 0.857 & & 0.107 & & & & & & & \\
\hline $\begin{array}{l}\text { Difference: } \\
\text { d14 p.o.-pre-op }\end{array}$ & & & & & & & & & & & & \\
\hline Median & 0.9 & -0.5 & -1.3 & -0.7 & -206 & -325 & & & & & & \\
\hline 1st/3rd quartile & $-0.2 / 3.7$ & $-0.9 / 0.1$ & $-8.1 / 3.3$ & $-1.7 / 0.7$ & $-418 / 1$ & $-591 /-138$ & & & & & & \\
\hline $\operatorname{Min} / \operatorname{Max}$ & $-2.2 / 7.4$ & $-2.6 / 2.4$ & $-11.7 / 11.5$ & $-3.3 / 1.4$ & $-943 / 320$ & $-1167 / 302$ & & & & & & \\
\hline $\mathrm{p}$ to group $\mathrm{N}$ & 0.021 & & 0.561 & & 0.403 & & & & & & & \\
\hline Progression curve & $\mathrm{p}=0.851$ & & $\mathrm{p}=0.261$ & & $\mathrm{p}=0.851$ & & & & & & & \\
\hline
\end{tabular}

For each factor analysis, the p-value for the comparison of the progression curves is indicated. For abbreviations see legend of Table II.

\section{Discussion}

The combination of surgical removal and antibody infusion seems to be an ideal treatment against large solid tumors, in particular for the prevention of minimal residual disease and spreading of metastases. In a pilot study we have evaluated such an approach for the treatment of gastric cancer. The monoclonal antibody SC-1 is highly specific for signet ring 
cell stomach carcinomas and induces by itself apoptosis in these cells. The SC-1 antibody is of the IgM isotype and was derived from human B-cells, most likely of the B1 subset. Thus, the antibody should be well tolerated after infusion and the induction of immunoglobulins against the injected antibody is highly improbable.

In this study, we evaluated the effect of the antibody treatment on the immune system, either directly after infusion or 7 and 14 days after surgery and compared the results with control group, in which stomach carcinomas were removed without prior antibody treatment.

We observed a slight increase of TNF- $\alpha$ in the blood serum after the antibody infusion, however the increased levels were still within the range observed in healthy individuals. One possible cause for this increase could be traces of endotoxin left in the antibody preparation, although all batches had been tested to be endotoxin-free. Another possibility, in particular considering the extremely small increase, could be psychosomatic effects of the infusion. The TNF- $\alpha$ levels stayed elevated after surgery, however again at an absolutely low level and not significantly different to patients without antibody treatment.

The most prominent effect we observed in the study was the lymphocytopenia after surgery. This effect was clearly caused by the surgery, since there was no difference to the patients without antibody treatment. Lymphocytopenia is quite normal after surgery and has been repeatedly described in the literature (19-23). The same applies to the drop of the CD3 ${ }^{+}$T-cells (19-23), which we observed only in the SC-1 treated patients. This drop seems to be due to a reduction in $\mathrm{CD}^{+}$cytotoxic T-cells, while the $\mathrm{CD}^{+}$helper T-cells remained virtually unchanged.

This is in contrast to most published data on postoperative lymphocytopenia, where the reduction in T-cells is mainly due to a reduction in $\mathrm{CD}^{+}$helper T-cells (19-23). Now we cannot offer a rational explanation for this effect, however, we want to emphasize that the reduced values are still in the range observed in healthy individuals. Inversely to the drop of $\mathrm{CD}^{+} \mathrm{T}$-cells we saw an increase of CD19+ B-cells in SC-1 treated patients 7 days post surgery. Since we measured the relative proportion of these cells among the lymphocytes, the increase in $\mathrm{CD} 19^{+} \mathrm{B}$-cells could simply be the compensation for the drop of $\mathrm{CD}^{+} \mathrm{T}$-cells (since all populations have to add up to $100 \%$, the reduction in one percentage increases the other percentages therefore). Conversely, we cannot exclude the possibility of a real increase of CD19+ B-cells with consequential reduction in $\mathrm{CD}^{+} \mathrm{T}$-cells.

Overall, we could not detect any clinically relevant difference between the SC-1 treated and untreated patients. The SC-1 treatment did not cause any overt inflammation or activation of the systemic immune system nor any serious immune suppression, exposing the patient to the risk of opportunistic infections after surgery. Thus, from an immunological point of view the application of the SC-1 antibody appears to be very safe.

Gastric cancer is one of the most common forms of cancer, with nearly 200,000 new cases each year in Europe (24). Curative resection of the stomach is the conventional treatment, however, the survival rates are very poor: about $80 \%$ after one year and around $45 \%$ after 5 years (25). Relapse of the tumor, originating from microscopically small metastases, are the main reason for this unsatisfactory prognosis. Thus, fighting these micrometastases or minimal residual disease using adjuvant or neoadjuvant protocols should improve the survival rate. Chemotherapy, radiotherapy or the combination of both have been used as adjuvant therapy to improve the survival rate (26). In most cases, the effect was minimal except for a recently published protocol achieving a $50 \% 3$-year survial rate compared to $41 \%$ without the adjuvant treatment.

This was achieved by a combination of chemo- (fluorouracil, leucovorin) and radiotherapy (45 Gy administered over 5 weeks) with the usual side effects of such a treatment (27). In addition, such a treatment is in no way specific for the tumor cells and affects other non-transformed rapidly dividing cells like leucocytes. Thus, a tumor specific adjuvant treatment would be much more effective and with much less side effects. To our knowledge, the application of SC-1 is the first tumor specific neoadjuvant therapy for gastric cancer tested in a clinical setting. While the effectiveness of the therapy is still under evaluation, clinical data and the data presented here clearly show that this neoadjuvant treatment has only minimal side effects, clearly much less than all chemo- or radiotherapy protocols. In the pilot study only in few cases side effects, such as fever during the antibody administration, were observed, however, all side effects were of grade 1 or 2 according to WHO classification and were completely reversible. Furthermore, this was the first case of using a human monoclonal natural $\operatorname{IgM}$ antibody for cancer therapy. So far, only one other clinical study has been performed using human IgM in cancer therapy. In that case, an anti-melanoma antibody specific for the ganglioside GM3 was applied in much higher doses (1X $960 \mathrm{mg}$ to $3 \mathrm{X}$ $1,920 \mathrm{mg}$ ) than in this study (20-30 mg) again without any adverse side effects (28). Thus, it seems that the application of IgM in tumor therapy is extremely safe and well tolerated by the patients.

\section{References}

1. Von Mehren M, Adams GP and Weiner LM: Monoclonal antibody therapy for cancer. Annu Rev Med 54: 343-369, 2003.

2. Waldmann TA, Levy R and Coller BS: Emerging therapies: spectrum of applications of monoclonal antibody therapy. Hematology (Am Soc Hematol Educ Program): 394-408, 2000.

3. Bruno L, Nesi G, Montinaro F, Carassale G, Boddi V, Bechi P and Cortesini C: Clinicopathologic characteristics and outcome indicators in node-negative gastric cancer. J Surg Oncol 74: 30-32, 2000

4. Fukagawa T, Sasako M, Mann GB, Sano T, Katai H, Maruyama K, Nakanishi Y and Shimoda T: Immunohistochemically detected micrometastases of the lymph nodes in patients with gastric carcinoma. Cancer 92: 753-760, 2001.

5. Kell MR, Winter DC, O'Sullivan GC, Shanahan F and Redmond HP: Biological behaviour and clinical implications of micrometastases. Br J Surg 87: 1629-1639, 2000.

6. Vollmers HP, Zimmermann U, Krenn V, Timmermann W, Illert B, Hensel F, Hermann R, Thiede A, Wilhelm M, RuckleLanz H, Reindl L and Muller-Hermelink HK: Adjuvant therapy for gastric adenocarcinoma with the apoptosis-inducing human monoclonal antibody SC-1: first clinical and histopathological results. Oncol Rep 5: 549-552, 1998.

7. Vollmers HP, O'Connor R, Muller J, Kirchner T and MullerHermelink HK: SC-1, a functional human monoclonal antibody against autologous stomach carcinoma cells. Cancer Res 49: 2471-2476, 1989. 
8. Hensel F, Hermann R, Schubert C, Abe N, Schmidt K, Franke A, Shevchenko A, Mann M, Muller-Hermelink HK and Vollmers HP: Characterization of glycosylphosphatidylinositollinked molecule CD55/decay-accelerating factor as the receptor for antibody SC-1-induced apoptosis. Cancer Res 59: 5299-5306, 1999.

9. Vollmers HP, Dammrich J, Ribbert H, Wozniak E and MullerHermelink HK: Apoptosis of stomach carcinoma cells induced by a human monoclonal antibody. Cancer 76: 550-558, 1995.

10. Vollmers HP, Hensel F, Hermann R, Dammrich J, Wozniak E, Gessner P, Herrmann B, Zimmermann U and MullerHermelink HK: Tumor-specific apoptosis induced by the human monoclonal antibody SC-1: a new therapeutical approach for stomach cancer. Oncol Rep 5: 35-40, 1998.

11. Ditschkowski M, Kreuzfelder E, Rebmann V, Ferencik S, Majetschak M, Schmid EN, Obertacke U, Hirche H, Schade UF and Grosse-Wilde H: HLA-DR expression and soluble HLA-DR levels in septic patients after trauma. Ann Surg 229: 246-254, 1999.

12. Syk I, Andreasson A, Truedsson L and Risberg B: Postoperative down-regulation of MHC class II antigen on monocytes does not differ between open and endovascular repair of aortic aneurysms. Eur J Surg 165: 1035-1042, 1999.

13. Duch DS, Bowers SW, Woolf JH and Nichol CA: Biopterin cofactor biosynthesis: GTP cyclohydrolase, neopterin and biopterin in tissues and body fluids of mammalian species. Life Sci 35: 1895-1901, 1984.

14. Huber C, Batchelor JR, Fuchs D, Hausen A, Lang A, Niederwieser D, Reibnegger G, Swetly P, Troppmair J and Wachter H: Immune response-associated production of neopterin. Release from macrophages primarily under control of interferongamma. J Exp Med 160: 310-316, 1984.

15. Sobin LH, Wittekind $\mathrm{C}$ and International Union Against Cancer: TNM classification of malignant tumours. 6th edition. New York, Wiley-Liss, 2002.

16. R Development Core Team: R: A language and environment for statistical computing. URL http://www.R-project.org Vienna, Austria, R Foundation for Statistical Computing, 2004.

17. Krauth J: Non-parametric formalizations for evaluating of time effect curves. Biom Z 15: 557-566, 1973.

18. Tukey JW: Exploratory Data Analysis. Addison-Wesley, 1977.
19. Cristaldi M, Rovati M, Elli M, Gerlinzani S, Lesma A, Balzarotti L and Taschieri AM: Lymphocytic subpopulation changes after open and laparoscopic cholecystectomy: a prospective and comparative study on 38 patients. Surg Laparosc Endosc 7: 255-261, 1997.

20. Fosse E, Opdahl H, Aakvaag A, Svennevig JL and Sunde S: White blood cell populations in patients undergoing major vascular surgery. Scand J Thorac Cardiovasc Surg 19: 247-252, 1985.

21. Garcia-Valdecasas JC, Martinez A, Lopoez-Boado MA, De Lacy AM, Cugat E, Grande L, Fuster J, Visa J and Pera C: Peripheral blood lymphocyte changes after benign gastrointestinal surgery. Role of thymostimulin in reversing the postoperative immunodepression seen in humans. Hepatogastroenterology 35: 219-222, 1988.

22. Hiesmayr MJ, Spittler A, Lassnigg A, Berger R, Laufer G, Kocher A, Artemiou O, Boltz-Nitulescu G and Roth E: Alterations in the number of circulating leucocytes, phenotype of monocyte and cytokine production in patients undergoing cardiothoracic surgery. Clin Exp Immunol 115: 315-323, 1999.

23. Lennard TW, Shenton BK, Borzotta A, Donnelly PK, White M, Gerrie LM, Proud G and Taylor RM: The influence of surgical operations on components of the human immune system. Br J Surg 72: 771-776, 1985.

24. Parkin DM, Bray F, Ferlay J and Pisani P: Global cancer statistics, 2002. CA Cancer J Clin 55: 74-108, 2005.

25. Siewert JR, Bottcher K, Stein HJ and Roder JD: Relevant prognostic factors in gastric cancer: ten-year results of the German Gastric Cancer Study. Ann Surg 228: 449-461, 1998.

26. Earle CC, Maroun J and Zuraw L: Neoadjuvant or adjuvant therapy for resectable gastric cancer? A practice guideline. Can J Surg 45: 438-446, 2002

27. MacDonald JS, Smalley SR, Benedetti J, Hundahl SA, Estes NC, Stemmermann GN, Haller DG, Ajani JA, Gunderson LL, Jessup JM and Martenson JA: Chemoradiotherapy after surgery compared with surgery alone for adenocarcinoma of the stomach or gastroesophageal junction. N Engl J Med 345: 725730, 2001.

28. Irie RF, Ollila DW, O'Day S and Morton DL: Phase I pilot clinical trial of human IgM monoclonal antibody to ganglioside GM3 in patients with metastatic melanoma. Cancer Immunol Immunother 53: 110-117, 2004. 